\title{
ELECTROMAGNETIC STRUCTURE FUNCTIONS OF NUCLEONS IN THE REGION OF VERY SMALL $X$
}

\author{
E.V. BUGAEV \\ Institute for Nuclear Research of the Russian Academy of Sciences, \\ 7a, 60th October Anniversary prospect, Moscow 117312, Russia \\ B.V. MANGAZEEV† \\ Irkutsk State University, 1, Karl Marx Street, Irkutsk 664003, Russia
}

A two component model describing the electromagnetic nucleon structure functions in the low-x region, based on generalized vector dominance and color dipole approaches is briefly described.

\section{Introduction}

A starting point of the present model of deep inelastic lepton-nucleon scattering is generalized vector dominance (GVD) idea [1], the main ingredient of which is a double dispersion relation for the total cross section for the process $\gamma^{*} p \rightarrow X$,

$$
\sigma\left(s, Q^{2}\right)=\sum_{q} \int \frac{d M^{2}}{M^{2}+Q^{2}} \int \frac{d M^{\prime 2}}{M^{\prime 2}+Q^{2}} \rho\left(s, M^{2}, M^{\prime 2}\right) \frac{1}{s} A_{q q \rightarrow p}\left(s, M^{2}, M^{\prime 2}\right) .
$$

Here, $\mathrm{M}$ and $\mathrm{M}^{\prime}$ are invariant masses of the incoming and outgouing $q q$-pair in $\gamma^{*} p$ elastic amplitude $\mathrm{A}$.

It is assumed here that at high energies the virtual photon $\gamma^{*}$ fluctuates in a hadronic system ( $q q$-pair, to the leading order) well before the interaction with the target nucleon and, at the second step, $q q$-pair interacts with the target. Later, in nineties [2-3], the relation (1) was used, in diagonal approximation, i.e., with $\rho \sim \delta\left(M^{2}-M^{\prime 2}\right)$, for a separation of the "soft" and "hard" interactions, and the soft part was described by the Vector Dominance Model (VDM).

The present two-component model of deep inelastic scattering differs (in a description of the soft part) from those developed in [2-3] in two respects: we do not use diagonal approximation and, most important, we do not limit ourselves by accounting of low mass vector meson $(\rho)$ and, instead, take into account $\rho$-family (containing, except of $\rho$, the radial excitations $\rho$ ', $\rho$ ', ,...). The use of the real mass spectrum of vector mesons is more natural than the introduction, in [3], the additive quark model component and, besides, allows introducing the non-diagonal transitions (and checking the importance of them).

\section{A two component model}

In our previous papers [4], [5] we formulated the two-component model of electromagnetic structure functions of the nucleon. The nonperturbative (soft) component of the structure functions is described by the off-diagonal GVD with vector mesons having properties of usual hadrons. It was shown in [4] that the approach of the off-diagonal GVD alone cannot describe the experimental data if the destructive interference effects and corresponding cancellations of $\mathrm{VN} \rightarrow \mathrm{V}^{\prime} \mathrm{N}$ amplitudes inside of GVD sums are small (and they are really small if the vector mesons in the tower have the properties of usual hadrons). It was shown, as a result, that two modifications of the off-diagonal GVD scheme are needed: 1) cut-off factors reducing the probability of initial $\gamma-\mathrm{V}$ transitions must be introduced and 2) a "hard component" must be

\footnotetext{
$\dagger$ Work partially supported by the program "Development of Scientific Potential in Higher Schools of Russia" (project 2.2.1.1/1483, 2.1.1/1539)".
} 
added to describe the perturbative QCD part of the total process of the virtual photon-nucleon interaction. For a calculation of the cut-off factor we use the following basic assumption: an interaction of the initial qq-pair with the nucleon is meson dominated if (and only if) the transverse momenta of pair's quarks are not too high; only in this case are confinement forces effective. It can be easily shown that the relative part of pair's phase volume for pairs with quarks having transverse momenta, $\mathrm{p}^{\mathrm{T}}$, in the limits $\left(\mathrm{m}_{\mathrm{q}} \div p_{\max }^{T}\right)$ is given, approximately, by $\eta \approx 3\left(p_{\max }^{T} / M_{q \tau}\right)^{2}$, if $M_{q q}^{2}>>\left(p_{\max }^{T}\right)^{2}$. By definition, this value is just the required cut-off factor. Here, the value $p_{\max }^{T}$ is the model parameter. The average transverse size of the qq-pair is, at not very large $\mathrm{Q}^{2}$, inversely proportional to $\mathrm{p}^{\mathrm{T}}$. From comparison with experimental data on $\mathrm{F}_{2}$ we obtained for this parameter the value $0.385 \mathrm{GeV}$.

The simplest model of VN-scattering had been used [4], based on two-gluon exchange approximation and relativistic constituent quark model. Wave functions of the vector mesons were obtained from a solution of the Bethe-Salpeter (BS) equation using quasipotential formalism in the light-front form. The kernel of the BS-equation has the confining term of the harmonic oscillator type. The vector meson mass spectrum is of the form $m_{n}^{2}=m_{\rho}^{2}(1+2 n)$, for the $\rho$-meson family (only this family has been taken into account). For the hard component, we used the color dipole model and the parameterizations of the dipole cross section $\sigma\left(r_{\perp}, s\right)$ (perturbative QCD part) from the work by J. Forshaw et al [6].

As one can see from fig.1, there is rather good agreement of the model predictions with the available data on the electromagnetic structure function $\mathrm{F}_{2}$ in the region $\mathrm{x}<0.05$ and $\mathrm{Q}^{2}<10^{2}$ $\mathrm{GeV}^{2}$. The relative contribution in $\mathrm{F}_{2}$ of the soft (GVD) component strongly depends on the values of the kinematic variables $\mathrm{x}, \mathrm{Q}^{2}$. At not very small $\mathrm{x}\left(\mathrm{x} \sim 10^{-1} \div 10^{-2}\right)$ the contribution of the soft component is dominant up to $\mathrm{Q}^{2} \sim 10^{2} \mathrm{GeV}^{2}$. With a decrease of the $\mathrm{x}$-value the interval of $\mathrm{Q}^{2}$ in which soft component is dominant is reduced. For example, at $\mathrm{x} \sim 10^{-4}$ the soft component is relatively large only in the region of very small $\mathrm{Q}^{2}, 0<\mathrm{Q}^{2}<1 \mathrm{GeV}^{2}$.

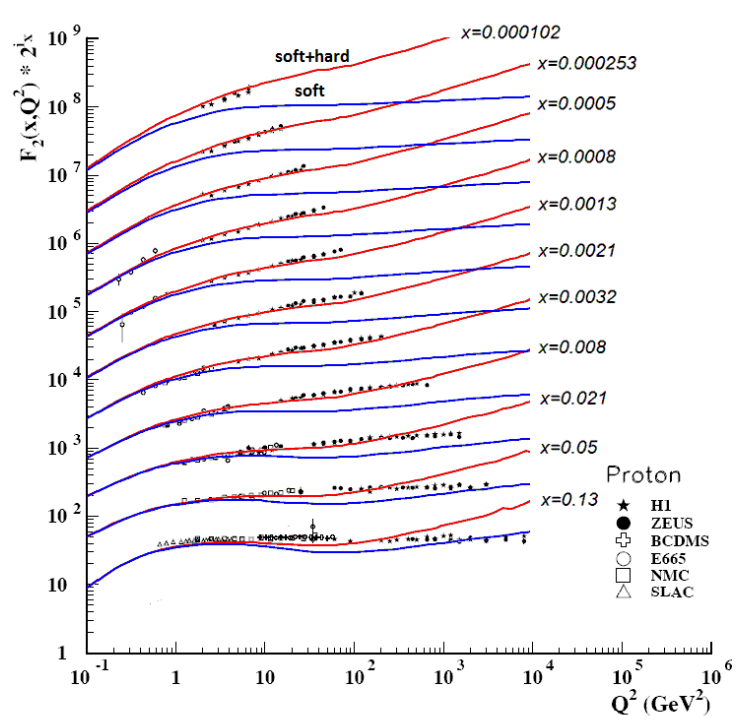

a
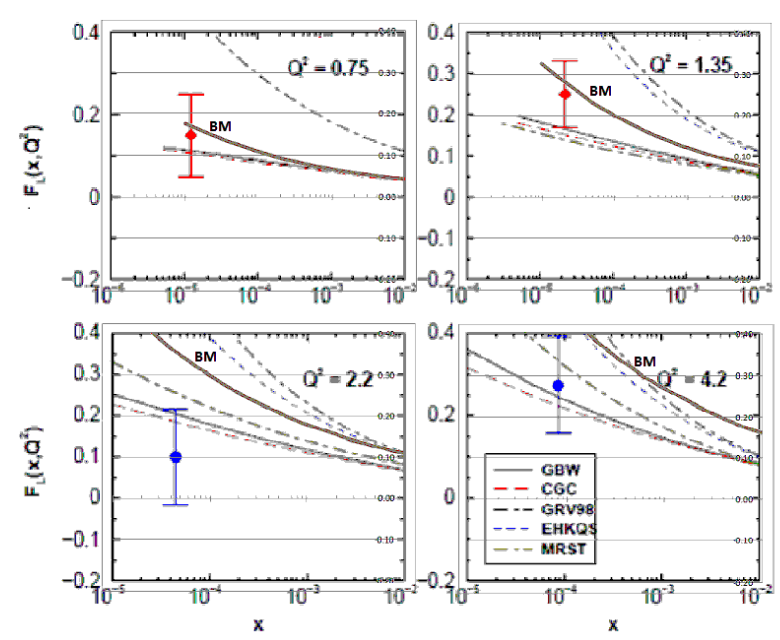

b

Figure 1. a) The proton electromagnetic structure function $F_{2}$ measured in experiments $H 1$, ZEUS, BCDMS, E665, NMC, SLAC [7]. Data of each bin of fixed $x$ has been multiplied by $2^{i}$, where $i$ is the odd number of the bin, ranging from $i=7(x=0.13)$ to $i=27(x=0.000102)$. The red lines are predictions of the present model (soft+hard), blue lines are the contributions of the soft (GVD). b) The results for $F_{L}$ as a function of $x$ at fixed $Q^{2}$. The data are taken from $H 1$ Collaboration. Solid brown curves $(\mathrm{BM})$ are results of the present model. Other curves are predictions of different theoretical models (see [8]). 


\section{References}

V.N. Gribov, Sov. Phys. JETP. 30, 709 (1970).

E. Gotsman et al, Eur. Phys. J. C5, 303 (1998).

A.D. Martin et al, Eur. Phys. J. C7, 643 (1999).

E. Bugaev, B. Mangazeev and Y. Shlepin, hep-ph/9912384(1999).

E. Bugaev and Y. Shlepin, Phys. Rev. D67, 034027 (2003).

J. Forshaw, G. Kerley and G. Shaw, Phys. Rev. D60, 074012 (1999).

http://pdg.lbl.gov/2007/reviews/strucfunfigrpp.pdf (2007).

V.Gonsales, M.Machado, hep-ph/0406230 (2004). 